\title{
Magnetic Control of the Earthquakes
}

\author{
A. L. Buchachenko ${ }^{1,2,3,4}$ \\ ${ }^{1}$ Institute of Chemical Physics, Russian Academy of Sciences, Moscow, Russia \\ ${ }^{2}$ Institute of Problems of Chemical Physics, Russian Academy of Sciences, Chernogolovka, Russia \\ ${ }^{3}$ Scientific Center in Chernogolovka, Chernogolovka, Russia \\ ${ }^{4}$ Moscow State University, Moscow, Russia \\ Email: alb9397128@yandex.ru
}

How to cite this paper: Buchachenko, A.L. (2021) Magnetic Control of the Earthquakes. Open Journal of Earthquake Research, 10, 138-152. https://doi.org/10.4236/ojer.2021.104009

Received: September 22, 2021

Accepted: November 5, 2021

Published: November 8, 2021

Copyright ( 2021 by author(s) and Scientific Research Publishing Inc. This work is licensed under the Creative Commons Attribution International License (CC BY 4.0).

http://creativecommons.org/licenses/by/4.0/ (c) (i) Open Access

\begin{abstract}
Many observations reliably exhibit correlations between the magnetic perturbations and seismic responses, convincing that the magneto-seismicity is not a myth. Magnetic control of the earthquakes is based on physics of magneto-plasticity, the remarkable phenomenon, which implies generation of the electron spin pairs on the trapped dislocations, in which Coulomb interaction is switched off. Microwave irradiation at Zeeman frequencies in these pairs stimulates the motion of dislocations, inducing release of elastic energy into the safe plastic deformation. Magneto-seismic correlations unambiguously demonstrate that the earthquakes are indeed suppressed by low-frequency (wide and continuous spectrum from $\mathrm{Hz}$ to $\mathrm{MHz}$ ) microwaves The detailed mechanism of this phenomenon is discussed and experimental proofs are given in terms of magneto-plasticity as a feasible means to control earthquakes.
\end{abstract}

\section{Keywords}

Earthquake, Magneto-Plasticity, Dislocation, Microwaves, Spin Pairs

\section{Introduction}

Earthquake (EQ) is an inevitable event but its magnitude may be controlled. Keeping in mind that the rocks are heterogeneous at many scales, from its smallest atomic scale (crystal defects, impurities, dislocations) to the different interfaces (micro-cracks, interphase and grain boundaries) one can conclude that the EQ may be controlled only at the atomic scale. The author shares the key paradigm formulated by Sornette [1] that "it is not possible to get a reasonable description of an EQ if one forgets about the chemical processes occurring at the smallest scale". The preparation of an EQ and its triggering proceeds on the atom- 
ic level and, therefore, in order to control EQ it is reasonable to rely on the atomic scale. The purpose of the paper is to suggest magnetically sensitive chemical mechanism of the dislocation motion as a means to control earthquakes.

\section{Results and Discussion}

\subsection{Accumulation and Release of Elastic Energy}

Elastic energy in the EQ focus is known to be created by anisotropic deformation induced by tectonic stress in rocks; it is accumulated and stored mostly in the dislocations trapped by impurities in crystal lattice, by neighboring dislocations or interfaces. These trapped dislocations keep the great excess of elastic energy for a long time, until a threshold is reached at which it is suddenly and unpredictably released as an EQ. The major part of elastic energy is kept as internal energy of crystal, hidden in dislocations trapped on the atomic defects or ionic impurities; in solid state physics, it is known as a dislocation strengthening. This is a reason why elastic energy is long living. In order to release it, at least partly, it is necessary to wake up these trapped, sleeping dislocations, to free them; then their motion transforms dangerous elastic energy into the safe energy of plastic deformation.

There are three the most important means if not to empty but decrease supply of elastic energy in the EQ core at the atomic scale. The first is the acoustic waves, regardless of what is their origin (nuclear or usual explosions, impacts of meteorites, triggering by other earthquakes, etc.). The propagation of the waves in crystal modulates interatomic distances and induces detachment of trapped dislocations stimulating their motion. The second factor, which is intensively discussed, is the fluids emanating the Earth core. They are supposed to create local pressure in cracks stimulating their expanding or to function as a lubrication of micro-cracks facilitating sliding along the cracks or interfaces. But cracks are not communicative vessels; fluids are forced to penetrate rocks by diffusion through the interstitial positions or vacancies in crystal lattice. The fluids are not able to diffuse as a molecular species (for exception, perhaps, of helium): $\mathrm{H}_{2}$, $\mathrm{H}_{2} \mathrm{O}$ and other molecules dissociate and diffuse as atoms or, what is chemically more reliably, as the protons (if atoms lose electron), hydride ions (if atoms attach electron) or ions $\mathrm{O}^{-}, \mathrm{O}^{2-}$, etc. Moving in crystal lattice these ions perturb local atomic surrounding in the vicinity of trapped dislocations by modulation of inter-atomic distances, stimulating detachment of dislocations. This mechanism seems to be the most probable to explain the fluid effects; it is indeed similar to the acoustic stimulation of sleeping dislocations. Nevertheless, only magnetic interactions may be considered as a controlling factor; it is technically the most easily implemented. Now we will consider magneto-seismic effects, which seem to be reliably established and physically sensible.

\subsection{Magnetically Controlled Seismicity}

Magneto-seismicity is not a myth. There are many observations, which reliably 
exhibit correlations between magnetic perturbations and seismic response. These correlations are found both in natural magnetic perturbations (magnetic storms) and artificial exposure to electromagnetic irradiation of the earthquake core by discharges of magneto-hydrodynamic generator; some examples will be given below.

Figure 1 clearly demonstrates that the tectonic deformation $\varepsilon$ drastically increases after irradiation of the core by magneto-hydrodynamic generator (these experiments were carried out in Central Asia regions Garm and Bishkek). The rates of deformation were also increased by 10 - 20 times; thus, in Garm the deformation rate before irradiation was 2.42 (in generally accepted conventional units), while under irradiation the deformation rate (38.8) was almost 20 times higher [2] [3]. These observations actually demonstrate slow plastic deformation of the EQ core induced by electromagnetic pulses.

The correlation of the two events, magnetic storms and earthquakes, was analyzed by Guglielmi et al. [4]. It is based on the observation of these two events during 1973-2010 years and presented in Figure 2. Here the storm onset is taken as zero time, then the number of large magnitude EQ $(M \geq 5)$, that took place 60 min before the storm onset and $60 \mathrm{~min}$ after it, were determined. If these two events, storm and EQ, were independent, 405 analyzed earthquakes must be distributed almost equally. However, this expectation is not confirmed: 230 earthquakes occurred before the storm and only 173 after it. It means that the number of strong earthquakes with $M \geq 5$ decreases after the storm by more than $30 \%$, i.e. the EQ focuses partially lose their elastic energy. This 30\% decreasing means that the magnetic storm eliminates every third large magnitude EQ, transforming

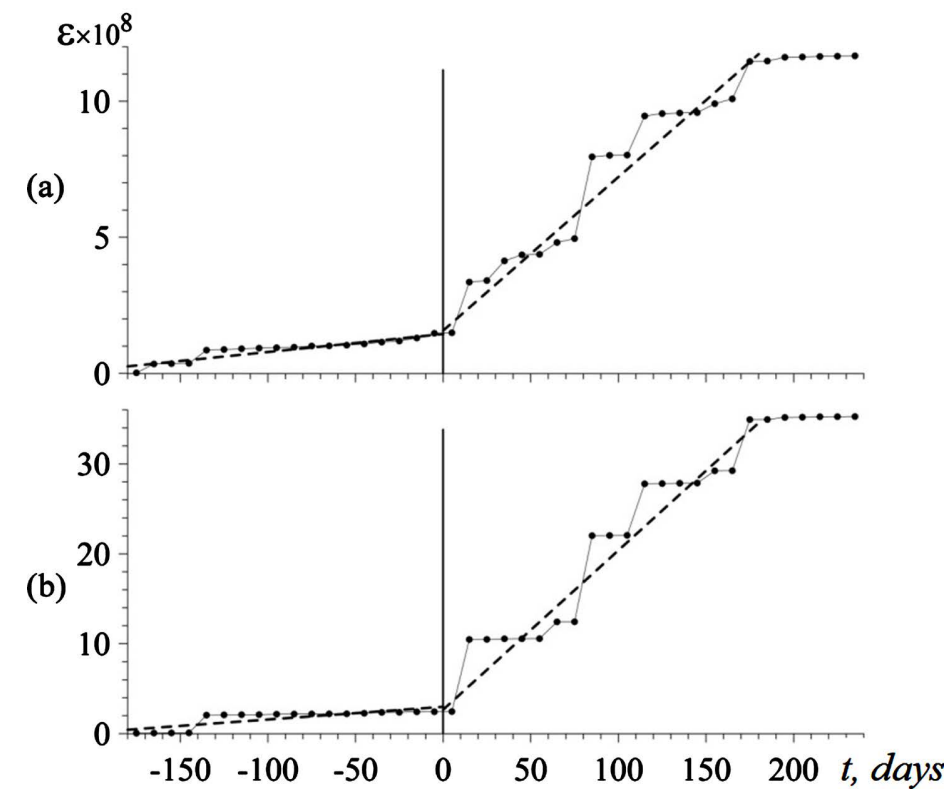

Figure 1. Total tectonic deformations $\varepsilon$ (a) and deformations of the upper layer $(b)$ before irradiation by electromagnetic pulses from magneto-hydrodynamic generators $(\mathrm{t}<$ 0 ) and after it $(t>0)$. The instant of pulses corresponds to $t=0$ (for details see [2] [3]). See different scales on the y-axis. 


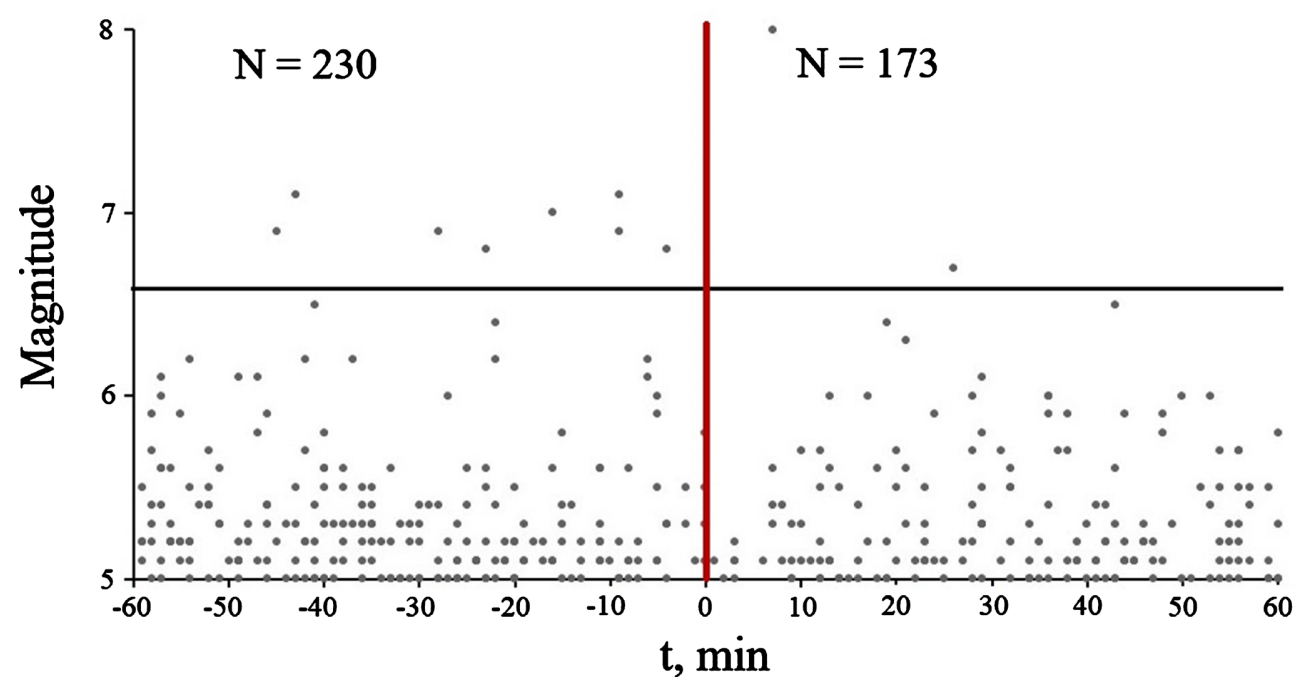

Figure 2. The number of EQ with magnitudes $M \geq 5$ in the time intervals of \pm 60 min with respect to the magnetic storm onset $(\mathrm{t}=0$, vertical red line).

it into the low magnitude EQ. The thick horizontal line in Figure 2 is drawn for the earthquakes with $M=6.6$; it is seen that 8 earthquakes with $M>6.6$ happened before the storm and only $2 \mathrm{EQs}$ after it. The effects of magnetic storms and electromagnetic initiation of slip were also discussed by Chelidze et al. [5] [6].

Figure 3 shows how the frequency of the powerful earthquakes $(M \geq 5)$ decreases after magnetic storms; it is evident that the effect of magnetic storms is significant [4]. It is statistically reliable conclusion that the magnetic storms suppress powerful earthquakes.

Extensive experiments with magneto-hydrodynamic generators for many years [7] have also detected correlation between magnetic and seismic events. For example, by measuring the number of EQ for 30 days before pulses of magneto-hydrodynamic generator $(m)$ and for 30 days after pulses $(n)$ it was shown that $m / n>1$ (about 1.15 - 1.45) for the large-magnitude EQ, but this ratio $m / n<$ 1 (about 0.8 - 0.9) for the low-magnitude EQs. At first glance, these effects seem to be enigmatic and contradictory. But these two effects are not independent: the suppression of a large-magnitude EQ means simultaneously its transformation into a small-amplitude EQ. The increasing number of weak earthquakes is a direct consequence of decreasing the number of the powerful earthquakes. Such a synchronism suggests that magnetic perturbations stimulate release of elastic energy of the earthquake focus by stimulation of trapped dislocations [8].

The relationship between the earthquakes and geomagnetic phenomena was established recently [9] [10]; the authors separated periods of geomagnetic activity into very quiet and extremely disturbed, and then correlated them with seismic activity. By analyzing the NEIC earthquake catalog of the US Geological Survey over a 20-year period, from 1980 to 1999 , it was shown that the planetary activity of earthquakes under quiet geomagnetic conditions is noticeably higher than the activity under disturbed conditions. In particular, the probability of the 

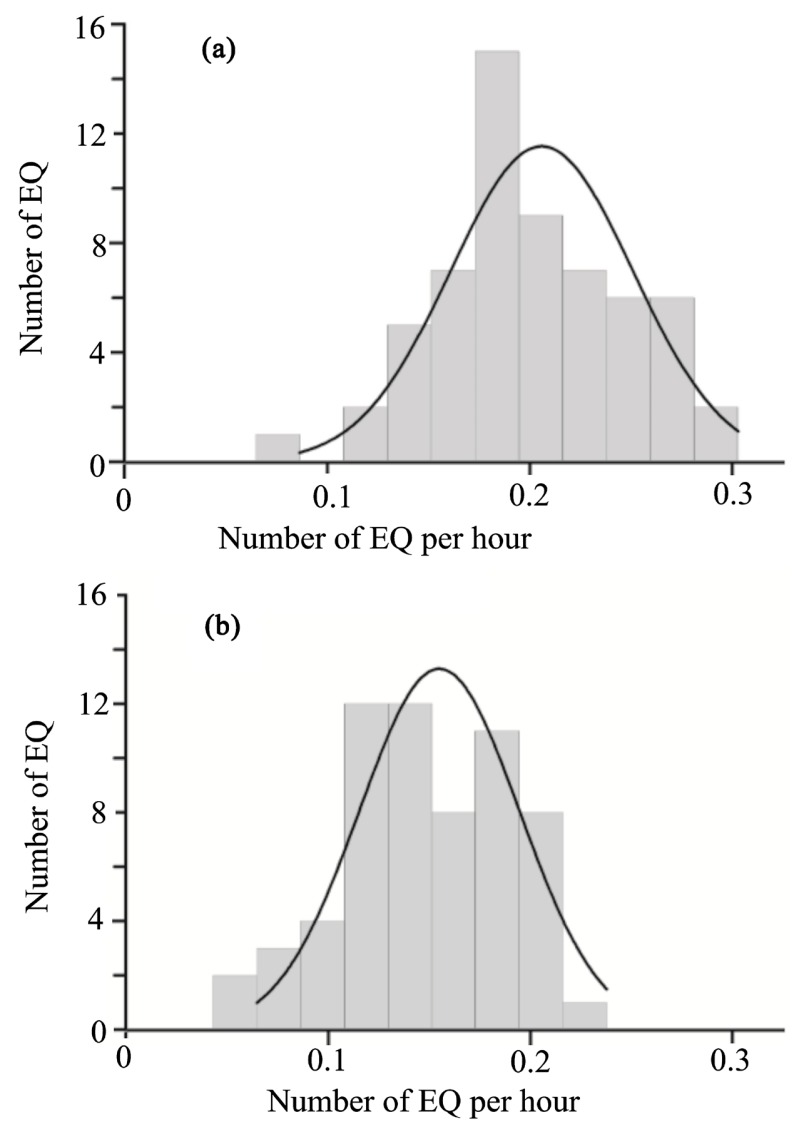

Figure 3. The distribution of the frequency earthquake appearance (number of EQ in 60 min interval) before magnetic storm (a) and after it (b). Smooth curves approximate normal distribution. These results were obtained by analysis of the large file of earthquakes for the time 1973-2010, using the method of epoch folding (digital synchronous detection method).

powerful earthquakes with magnitude $M \geq 8$ was shown to be twice higher in magnetically quiet days than that in the magnetically active days. This impressive result is in accordance with idea that geomagnetic activity stimulates release of elastic energy and unambiguously convinces the reliability of the magnetic control of seismicity.

The correlation between the strong natural magnetic storms and the seismic noise, accompanying these storms and characterized by pulses of displacements with duration of a few minutes [11], is in perfect agreement with magneto-hydrodynamic results mentioned above. The properties of the pulses do not depend on the weather conditions. The pulses are detected in the records from all seismic stations located on the continents. It is hypothesized that sharp changes in the electromagnetic field during a storm serve as a trigger for the release of energy accumulated in the Earth; the latter seems to induce displacement of rocks as a result of the motion of dislocations.

\subsection{Magneto-Plasticity as a Means to Control Dislocations}

Mechanical properties of diamagnetic crystals, such as hardness, deformation, 
plasticity are known to depend on the magnetic field [12] [13]. This phenomenon, called magneto-plasticity, seems to be enigmatic because crystals exhibiting this phenomenon have no magnetic components. The source of magneto-plasticity is firmly established [14] [15] to be dislocations: their mobility depends on the magnetic field and this dependence is a key to the magnetically dependent mechanics. It was suspected that some paramagnetic states of dislocations, vulnerable to the magnetic field, are formed in crystal, but the nature of these states was enigmatic. It was experimentally shown that the magneto-plasticity is hidden in the system dislocation + stopper. Because any contribution of the magnetic field into the energy budget of this system is irrelevant, one should suggest that the electron spin (angular momentum) plays a role in this phenomenon, inducing magnetic field controlled process. However, neither free dislocation, nor diamagnetic stopper (such as $\mathrm{Ca}^{2+}$ ion) carries any spin. Moreover, for the observation of magnetic effects, it is necessary to have two-spin system [16] [17].

The idea of the two-spin, magnetically sensitive electron pair in a stopper-captured, trapped dislocation was proposed [18] [19] [20]; it is illustrated by Scheme 1 for the particular case of dislocation trapped by $\mathrm{Ca}^{2+}$ ion in the $\mathrm{NaCl}$ crystal.

The scheme implies energy allowed electron transfer between partners (dislocation is presented by its anionic element $\mathrm{Cl}^{-}$ion; index $\mathrm{d}$ points out that the ion belongs to dislocation). It generates spin pair: each partner carries unpaired electron. It is in the singlet spin state $S$ because electron transfer does not change spin; the back electron transfer is spin allowed, so that the initial trapped dislocation may be restored. It is remarkable that in the spin pair Coulomb interaction is switched off, Coulomb trap disappears; the stopper does not hold dislocation, it is now free and starts new motion.

It is a first step to the magneto-plasticity. Magnetic field produces conversion of the spin pair from singlet spin state $S$ to triplet state $T_{0}$; it occurs as the spin dephasing by precession of individual spins as shown in Figure 4.

The arrangement of electron spins $S_{1}$ and $S_{2}$ in singlet and triplet states is presented in Figure 5.

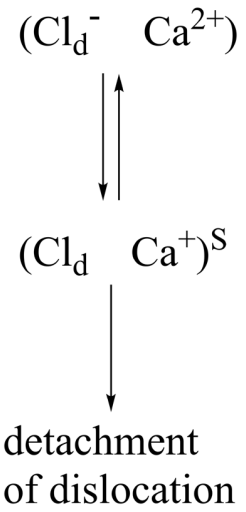

Scheme 1. Electron transfer in the system dislocation + stopper. 


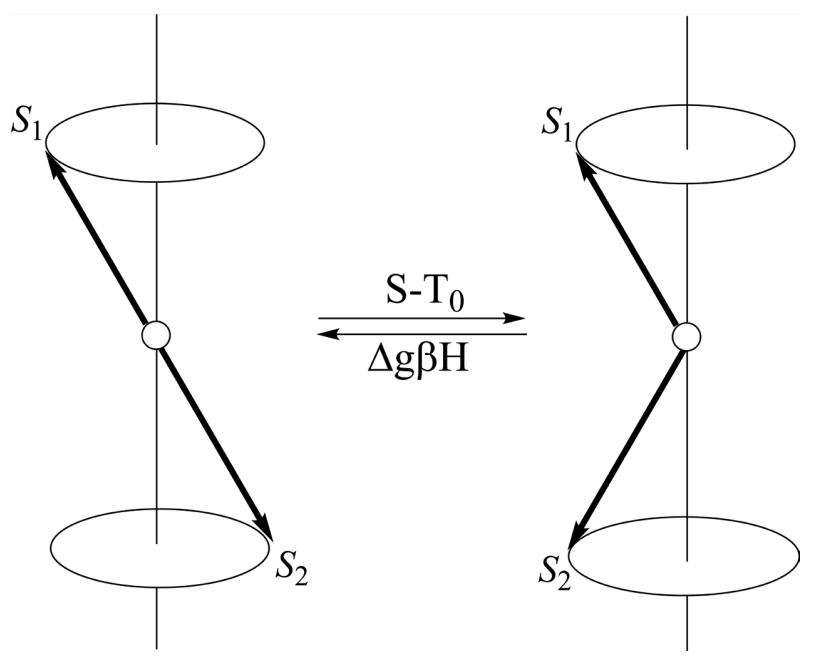

Figure 4. Spins $S_{1}$ and $S_{2}$ are located on the partners of the spin pair. They oscillate (precess) with Zeeman frequencies $\mathrm{g}_{1} \beta \mathrm{H}$ and $\mathrm{g}_{2} \beta \mathrm{H}$ respectively, where $\mathrm{g}_{1}$ and $\mathrm{g}_{2}$ are g-factors of partners. The rate of $\mathrm{S}-\mathrm{T}_{0}$ spin conversion is the difference of these Zeeman frequencies $\Delta \mathrm{g} \beta \mathrm{H}$, where $\Delta \mathrm{g}=\mathrm{g}_{1}-\mathrm{g}_{2}$.

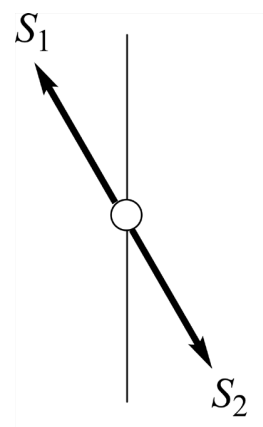

S

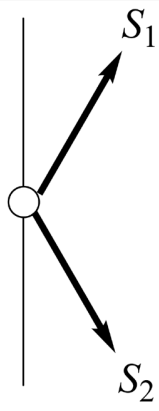

$\mathrm{T}_{0}$

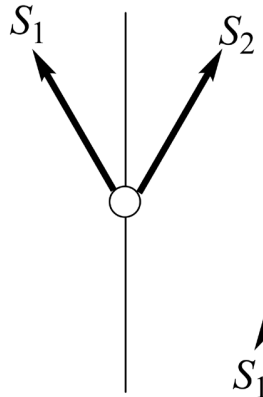

$\mathrm{T}_{+}$

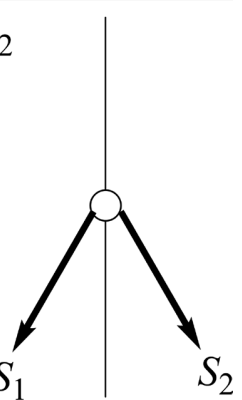

T.

Figure 5. Schematical presentation of the electron spins $S_{1}$ and $S_{2}$ in singlet and triplet states.

Magnetic field executes two functions: first, it produces reversible $S-T_{0}$ conversion with the rate $\Delta \mathrm{g} \beta \mathrm{H}$, as shown in Figure 4 and, second, it splits triplet state into the three states $\mathrm{T}_{0}, \mathrm{~T}_{+}$and $\mathrm{T}_{-}$; these states differ by spin projections: 0 , +1 and -1 respectively, as shown in Figure 6. Both functions are illustrated in Scheme 2 and Figure 6.

The second step to the magneto-plasticity and magnetic control of EQ is accomplished by microwaves. They convert $\mathrm{T}_{0}$ into $\mathrm{T}_{+}$and $\mathrm{T}_{-}$and populate these states of spin pair. It is extremely important to note that in contrast to the reversible $\mathrm{S}-\mathrm{T}_{0}$ spin conversion the transformation of $\mathrm{T}_{+}$and $\mathrm{T}_{-}$states into $\mathrm{S}$ state is strictly spin forbidden, so that the dislocation cannot return into the initial trapped state, it is doomed to leave these long living $\mathrm{T}_{+}$and $\mathrm{T}_{-}$ states of spin pair. This is a key point, where magnetic control of the EQ is implemented, where transformation of elastic energy into the energy of plastic deformation occurs. 


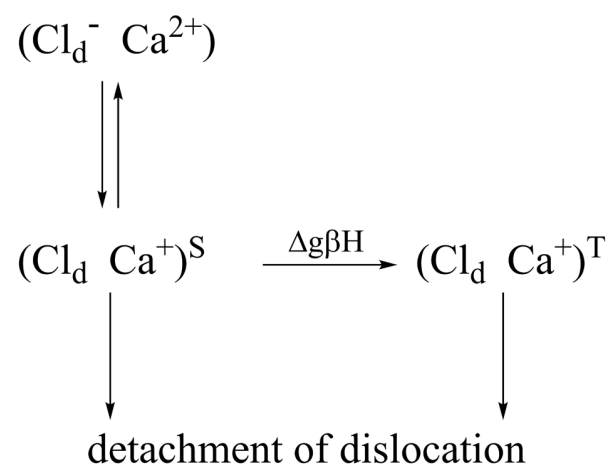

Scheme 2. Magnetic field converts singlet state $\mathrm{S}$ into the triplet state T.

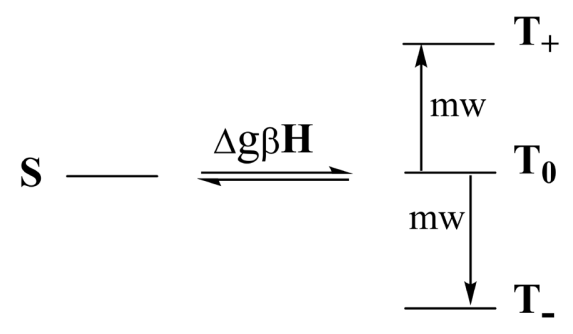

$\mathbf{H}=\mathbf{0} \quad \mathbf{H}>\mathbf{0}$

Figure 6. The scheme of Zeeman levels in zero $(\mathrm{H}=0)$ and high $(\mathrm{H}>0)$ magnetic field. Microwave irradiation mw induces transitions from $\mathrm{T}_{0}$ into $\mathrm{T}_{+}$and $\mathrm{T}_{-}$states.

\subsection{Microwave Reception}

Magnetic resonances at Zeeman frequencies $\mathrm{g} \beta \mathrm{H}$ in the spin pairs are the most reliable proof of the molecular concept of magneto-plasticity in terms of Schemes 1 and 2. Resonances were experimentally detected in many crystals as the acceleration of dislocations and increasing the path of their runs under irradiation by microwaves at Zeeman frequencies [21] [22]; it is indeed electron spin resonance, detected and measured by dislocation movement. As an example, Figure 7 demonstrates that the acceleration of dislocation may be almost doubled under microwave irradiation.

In the direct laboratory experiments when the series of strong electromagnetic pulses were applied to the rock samples it was shown that these pulses initiate acoustic emission as an indicator of mechanical instability, which stimulates slip of stressed samples [23]. The similar effects of acoustic emission triggered by electromagnetic irradiation of marble and other materials are described in the review [24].

The observation of microwave effects means that the microwave pumping controls spin evolution of spin pairs and the motion of dislocations. In other words, the pair is a microwave receiver; its mw pumping at Zeeman frequencies controls mechanical properties of crystals and solid state materials including rocks.

\subsection{Magnetic Transport of Dislocations}

Thus, magnetic fields, both permanent and electromagnetic, accelerate and cat- 
alyze the motion of dislocations and this magnetic catalysis creates magneto-plasticity as a physical phenomenon. Till now we have considered an ideal, individual spin pair on the trapped dislocation; its magnetic fate is presented in Figure 6. However, instead of a single, individual spin pair, in the crust of the EQ core there is a huge collection of trapped dislocations and spin pairs with great distribution of $\mathrm{G}$-factors and great scatter in local magnetic fields $\mathrm{H}$; it is schematically presented in Figure 8.

It means that instead of the single mw resonance there are multiple resonances, a huge stream of Zeeman transitions; the thick lines in Figure 8 symbolize this phenomenon.

The distribution in G-factors arises from the two sources. The first is that the even individual g-factor is itself anisotropic that is its Zeeman resonance depends on the orientation of the molecular axes with respect to direction of magnetic field. For any paramagnetic species there are three principle magnitudes $g_{x}$,

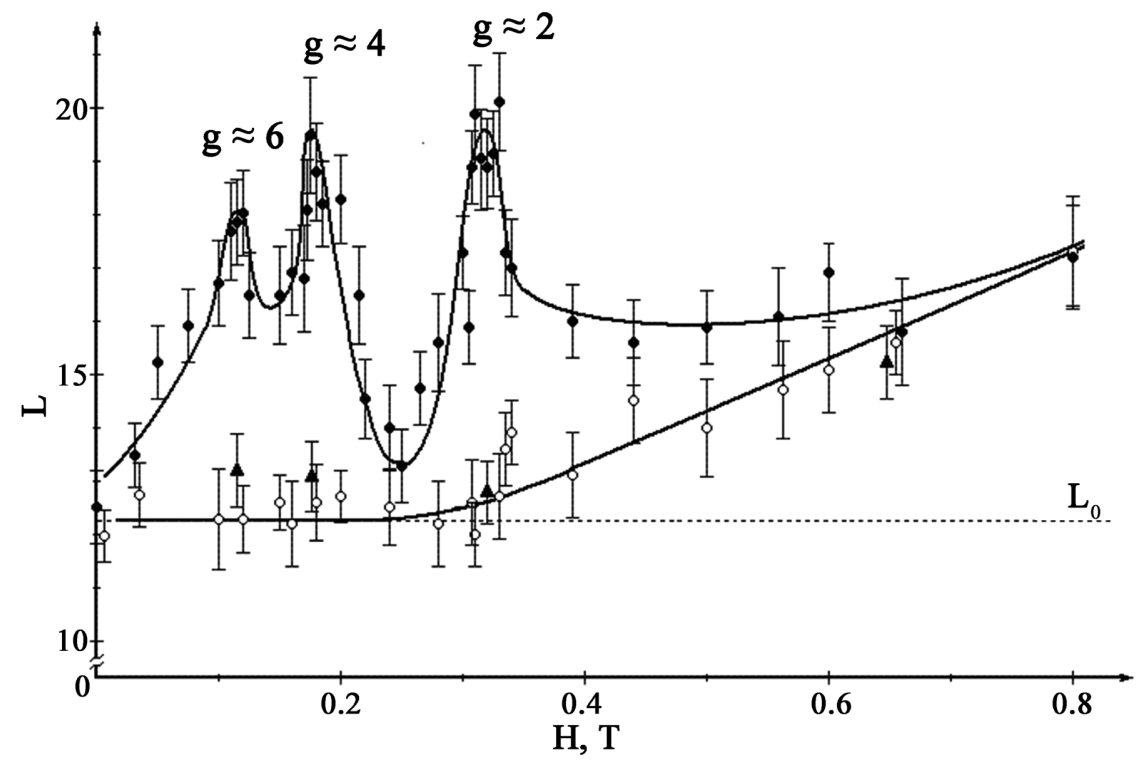

Figure 7. The run $\mathrm{L}$ of dislocations in the single $\mathrm{NaCl}$ crystal as a function of magnetic field $\mathrm{H}$. The resonance peaks occur at Zeeman frequencies $\mathrm{g}_{1} \beta \mathrm{H}(\mathrm{g} \approx 6), \mathrm{g}_{2} \beta \mathrm{H}(\mathrm{g} \approx 4)$, and $\mathrm{g}_{3} \beta \mathrm{H}(\mathrm{g} \approx 2)$. The lower curve indicates the runs without microwave irradiation; $\mathrm{L}_{0}$ is the run in the Earth magnetic field.

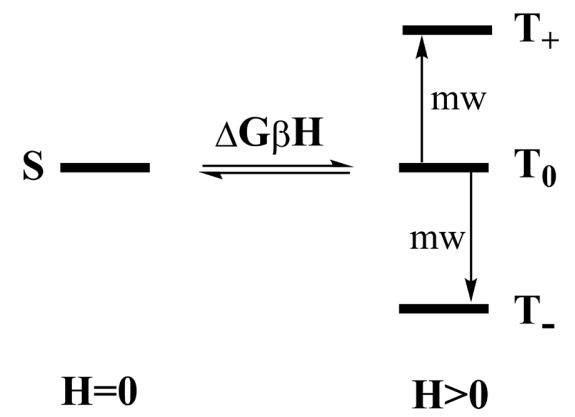

Figure 8. The scheme of Zeeman energy levels and spin transitions for the collection of pairs with different $\mathrm{g}$-factors $\mathrm{G}$ in varying magnetic fields $\mathrm{H}$. 
$\mathrm{g}_{\mathrm{y}}$, and $\mathrm{g}_{\mathrm{z}}$ which correspond to $\mathrm{g}$-factor, if magnetic field is oriented along the molecular axes $\mathrm{x}, \mathrm{y}$, or $\mathrm{z}$ respectively. In any arbitrary orientation of molecular axes g-factor falls in the broad range between $g_{x}$, $g_{y}$, and $g_{z}$. The other source of the distribution in G-factors is the variety of chemical composition of rocks, which implies a vast variety of chemically different trapped dislocations and spin pairs with a great collection of anisotropic g-factors.

The distribution in magnetic fields $\mathrm{H}$ also arises from the two sources. The first is induced by inhomogeneity of local magnetic fields in rocks (due to their different magnetic susceptibility, inclusion of ferromagnetic impurities, etc.). The other is the inconstancy of the Earth field, which is modulated and perturbed by magnetic storms and other emissions of ionosphere, by many fields emitted by products of technical civilization, etc.

Finally, we have a huge collection of Zeeman levels and the widest band of $\mathrm{mw}$ frequencies. Their dependence on the magnetic field is schematically shown in Figure 9(a). The transitions $T_{0}-T_{+}$and $T_{0}-T_{-}$fall in microwave region of $M H z$; more exactly, they are close to those which are Zeeman resonances around the Earth magnetic field. The great majority of these high frequency waves are hardly efficient in the stimulation of trapped dislocations due to their low penetrability in the rocks.

The levels shown in Figure 9(a) ignore dipolar interaction between unpaired electrons in spin pair. But it is not ignorable: magnetic field created by electron depends on the distance $r$ as $\beta / r^{3}$, where $\beta$ is the Bohr magneton. For instance, at $r=3 \AA \hat{~ i t ~ i s ~ a b o u t ~} 350 \mathrm{G}$ and comparable or even strongly exceeds Earth magnetic field (0.2 - $0.6 \mathrm{G})$. Like g-factor, dipolar interaction $B$ is the anisotropic tensor, its components $B_{x}, B_{y}$, and $B_{z}$ are different along the molecular axes of spin pair; moreover, they are of opposite signs and obey to relation $B_{x}+B_{y}+B_{z}=0$, so that
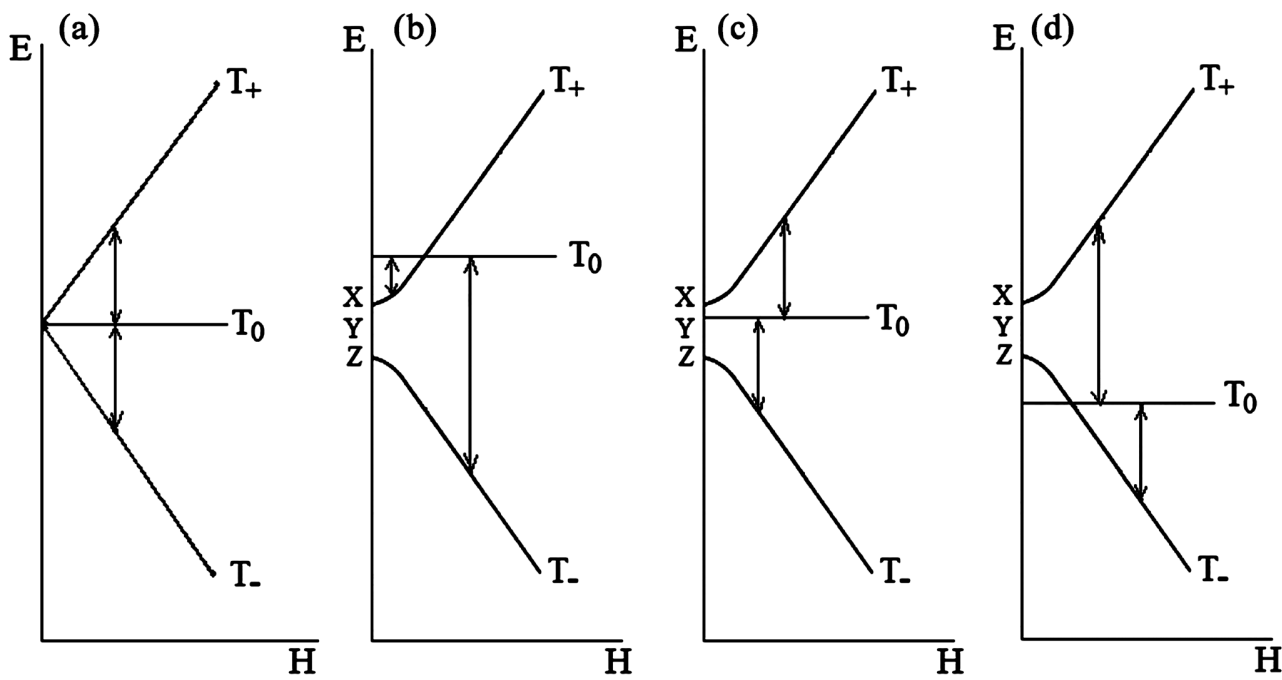

Figure 9. The scheme of Zeeman levels and resonance Zeeman transitions in spin pairs: $a$-dipolar interaction is absent; $b, c, d$-dipolar interaction is switched on; magnetic field is oriented along the molecular axes $\mathrm{x}(b), \mathrm{y}(c), \mathrm{z}(d)$. The $\mathrm{y}$-axis refers to the horizontal line $\mathrm{T}_{0}$. The energies are given in arbitrary units, arrows label Zeeman transitions. 
in any arbitrary orientation of molecular axes Zeeman transitions fall in the broad range between $B_{x}, B_{y}$, and $B_{z}$.

Again we have a huge collection of Zeeman levels and wide band of frequencies but now, when dipolar interaction is taken into account, their dependences on the magnetic field are different from that shown in Figure 9(a); these dependences are presented in Figures 9(b)-(d). They correspond to orientation of magnetic field along the molecular axes $\mathrm{x}, \mathrm{y}$, or $\mathrm{z}$ respectively. In zero magnetic field the spin states of the spin pair are denoted as $\mathrm{X}, \mathrm{Y}$, and $\mathrm{Z}$; it is well known in the electron spin resonance spectroscopy [25], where energy differences between states $\mathrm{X}, \mathrm{Y}$, and $\mathrm{Z}$ are named as zero field splitting. When magnetic field increases the states $\mathrm{X}, \mathrm{Y}$, and $\mathrm{Z}$ are transformed into $\mathrm{T}_{+}, \mathrm{T}_{0}$, and $\mathrm{T}_{-}$as shown in Figures 9 (b)-(d).

It is the most important that now, with taking into account of dipolar inter-electron interaction, the transitions $\mathrm{T}_{0}-\mathrm{T}_{+}$and $\mathrm{T}_{0}-\mathrm{T}_{-}$fall in the low frequency region from $\mathrm{Hz}$ to $\mathrm{kHz}$. It covers almost continuous band in the range of $\mathrm{kHz}$ frequencies; due to their penetrability in rocks these low frequency waves are efficient in the stimulating trapped dislocations. Namely these frequencies seem to be responsible for the magnetic stimulation of dislocations and magnetic control of the earthquakes described in Section 3. (Note also research of magnetic bias control system presented in reference [26]).

\subsection{Magneto-Plastic Effect: Experimental Observations}

Magnetic stimulation of trapped dislocations was described in many papers; numerous results are summarized in the review [13]. The example of high-frequency resonance acceleration of dislocations was shown in Figure 7; other examples of low-frequency resonances are seen in Figure 10 and Figure 11 . They are detected in the range of resonance frequencies $10^{3}-10^{6} \mathrm{~Hz}$ in Earth magnetic field in the $\mathrm{NaCl}$ crystals doped with $\mathrm{Ca}^{2+}$ ions [15] [27]. These multiple resonances belong to different orientations of molecular axes of spin pairs as described in

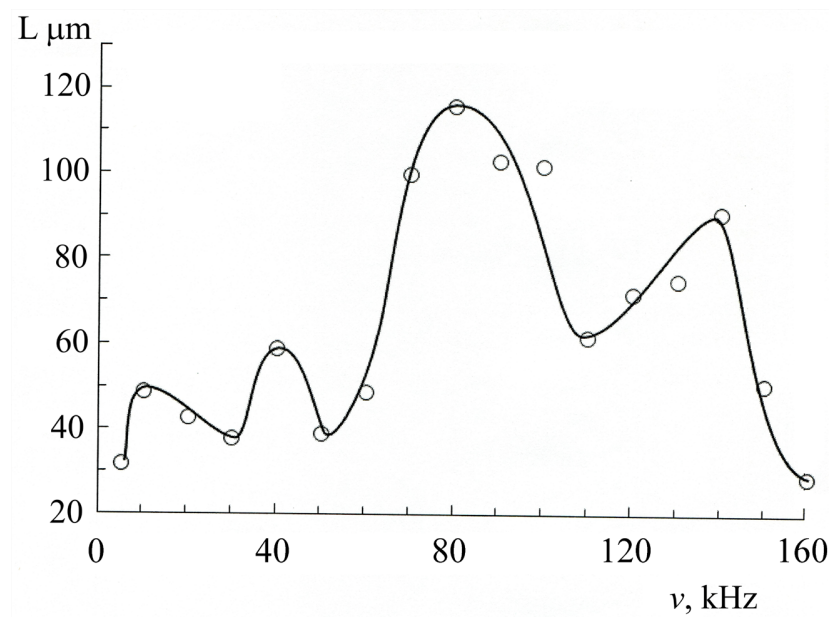

Figure 10. The trips $\mathrm{L}$ of dislocations in a single $\mathrm{NaCl}$ crystal at certain its orientation as a function of microwave pumping frequencies. 


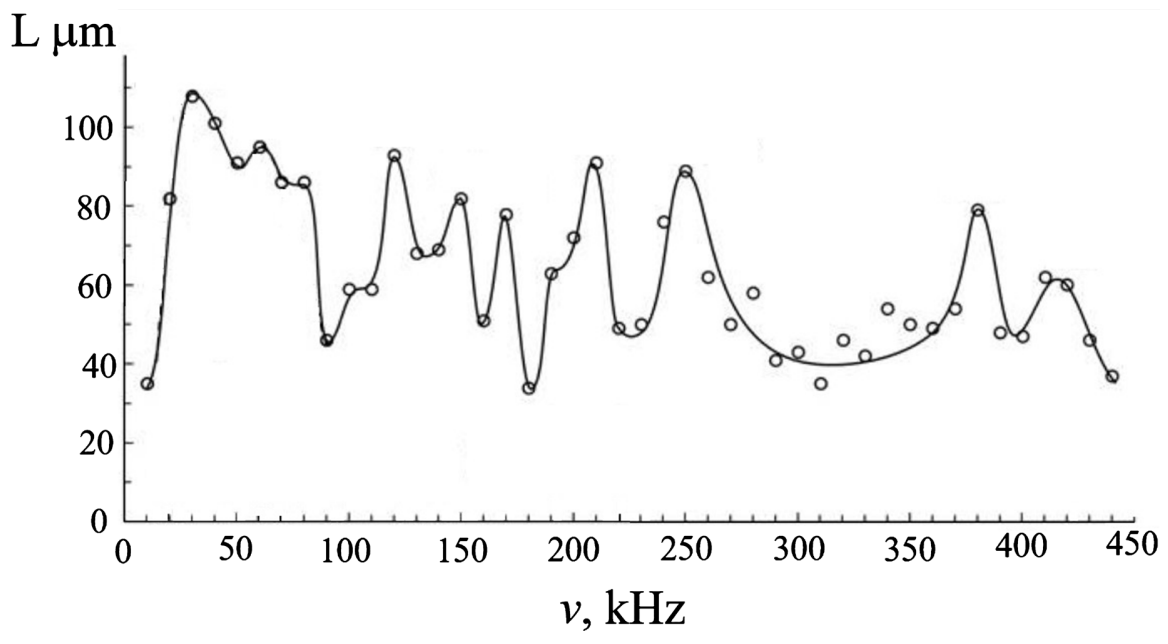

Figure 11. The trips $\mathrm{L}$ of dislocations in single $\mathrm{NaCl}$ crystal as a function of microwave frequencies with pulse pumping. Each point refers to the certain orientation of crystal.

previous section; the runs of dislocations under resonances increase by several times.

Figure 11 illustrates similar effects detected in Earth magnetic field with pulsed pumping [28] [29]. Again many resonances are observed; they belong to spin pairs in different orientations of molecular axes (due to anisotropy of $\mathrm{g}$-factors and dipolar interactions) and in different local magnetic fields. Note that they are at rather low frequencies and significantly accelerate dislocations. Of course, the runs of dislocations depend on the amplitude of microwave pumping. Thus, in the pulse regime with pulse duration $0.5 \mu$ s the runs are 30 and $70 \mu \mathrm{m}$ at the amplitudes of 0.05 and $0.10 \mathrm{G}$ respectively; the lifetimes of spin pairs fall in the limits of $10^{-5}-10^{-4} \mathrm{~s}$.

The acceleration of dislocations in Earth magnetic field under pulsed pumping was also detected in crystals of $\mathrm{ZnO}$, triglycine sulfate, and potassium hydrogen phthalate; all resonances were in the $\mathrm{kHz}$ region [30]. Evidently, the microwave induced magneto-plasticity is a general and universal phenomenon.

\section{Conclusions}

Experimental observations are in perfect agreement with the idea of magnetic control of EQ by stimulation of magneto-plasticity. It is conceivable to use magnetic control of dislocations as a means to release elastic stress in the earthquake focus to avoid a catastrophic earthquake by transforming it into a weak, small-magnitude one. Microwave irradiation transforms elasticity of diamagnetic solids into plasticity, controlling mobility of dislocations via magnetic interactions in the electron spin pairs on the dislocations; microwaves produce acceleration of dislocation and increase the path of their runs, providing release of elastic energy.

Microwave pumping on the resonance Zeeman frequencies (in the regime of magnetic resonances) is a solid proof of the physics of electron spin pairs and magneto-plasticity, as itself. The main contribution into macroscopic transfor- 
mation of elastic energy into the plastic flow is introduced by resonant microwaves. Namely these waves may be used to control mechanics of the earthquake focus.

It is worth noting that the magneto-seismicity is a new field of the Earth Sciences; it is natural that many questions remain unanswered, many details are not clear. What is important and worthy of confidence (at least, it cannot be ignored) is that the magneto-seismicity is illustrated by correlations between magnetic (magnetic storms, solar activity, power magnetic pulses of hydrodynamic generators) and seismic events; they indicate that the magnetic (microwave) effects on the earthquakes is not a myth, it is a means to transform large-magnitude earthquakes into the weak, low-magnitude events. Magnetic control is shown to be a double-faced phenomenon: it may devastate the highenergy focus suppressing large-magnitude earthquakes, but it may provoke low-magnitude, safe earthquakes, preventing accumulation of elastic energy and its releasing by catastrophic earthquakes.

\section{Acknowledgements}

The author thanks Professor Inoue Katsuya from the Hiroshima University for his encouraging and stimulating comments and Professors A. V. Gul'elmi and N. T. Tarasov for their critical and stimulating discussions. The special thanks are addressed to Professor Yoshifumi Tanimoto for his outstanding contribution into the Magneto-Science.

\section{Conflicts of Interest}

The author declares no conflicts of interest regarding the publication of this paper.

\section{References}

[1] Sornette, D. (1999) Earthquakes: From Chemical Alteration to Mechanical Rupture. Physics Reports, 313, 237-291. https://doi.org/10.1016/S0370-1573(98)00088-X

[2] Tarasov, N.T., Tarasova, N.V., Avagimov, P. and Zeigarnik, V.A. (1999) The Effect of High Energy Electromagnetic Pulses on Seismicity in Central Asia and Kazakhstan. Journal of Volcanology and Seismology, 4-5, 152-160.

[3] Tarasov, N.T. and Tarasova, N.V. (2004) Spatial-Temporal Structure of Seismicity of the North Tien Shan and Its Change under Effect of High Energy Electromagnetic Pulses. Annals of Geophysics, 47, 199-212. https://doi.org/10.4401/ag-3272

[4] Guglielmi, A.V., Lavrov, I.P. and Sobisevich, A.L. (2015) Storm Sudden Commencements and Earthquakes. Journal of Atmospheric and Solar-Terrestrial Physics, 1, 98-103. https://doi.org/10.12737/5694

[5] Chelidze, T.V., De Rubeis, T., Matcharashvili, R. and Tosi, P. (2002) Influence of Strong Electromagnetic Discharges on the Dynamics of Earthquakes Time Distribution at the Bishkek Test Area (Central Asia). Proceedings of ESC XXVIII General Assembly, Genova.

[6] Chelidze, T., Varamashvili, N., Devidze, M., Chelidze, Z., Chikladze, V. and Matcharashvili, T. (2002) Laboratory Study of Electromagnetic Initiation of Slip. Annals 
of Geophysics, 45, 587-598.

[7] Tarasov, N.T. and Tarasova, N.V. (2011) Influence of Electromagnetic Fields on the Seismotectonic Strain Rate; Relaxation and Active Monitoring of Elastic Stresses. Izvestiya, Physics of the Solid Earth, 47, 937-951.

https://doi.org/10.1134/S1069351311100120

[8] Buchachenko, A.L. (2014) Magneto-Plasticity and the Physics of Earthquakes. Can a Catastrophe Be Prevented? Physics-Uspekhi, 57, 92-98. https://doi.org/10.3367/UFNe.0184.201401e.0101

[9] Guglielmi, A.V., Klain, B.I. and Kurazhkovskaya, N.A. (2020) Earthquakes and Geomagnetic Disturbance. Solar-Terrestrial Physics, 6, 80-83.

https://doi.org/10.12737/stp-64202012

[10] Guglielmi, A.V. (2020) On the Relationship between Earthquakes and Geomagnetic Disturbances. Geophysical Research, 21, 78-83.

https://doi.org/10.12737/stp-64202012

[11] Sobolev, G.A., Zakrzhevskaya, N.A., Migunov, I.N., Sobolev, D.G. and Boiko, A.N. (2020) Effect of Magnetic Storms on the Low-Frequency Seismic Noise. Izvestiya, Physics of the Solid Earth, 56, 291-315. https://doi.org/10.1134/S106935132003009X

[12] Morgunov, R.B. (2004) Spin Micro-Mechanics in the Physics of Plasticity. Physics Uspekhi, 47, 131-153. https://doi.org/10.1070/PU2004v047n02ABEH001683

[13] Alshits, V.I., Darinskaya, E.V., Koldaeva, M.V., Kotowski, R.K., Petrzhik, E.A. and Tronczyk, P. (2017) Dislocation Kinetics in Nonmagnetic Crystals: A Look through a Magnetic Window. Physics Uspekhi, 60, 305-318. https://doi.org/10.3367/UFNe.2016.07.037869

[14] Alshits, V.I., Darinskaya, E.V., Koldaeva, M.V. and Petrzhik, E.A. (2008) In Dislocations in Solids. Vol. 14, Elsevier, Amsterdam, 333.

https://doi.org/10.1016/S1572-4859(07)00006-X

[15] Alshits, V.I., Darinskaya, E.V., Morozov, V.A., Kats, V.M. and Lukin, A.A. (2010) ESR in the Earth's Magnetic Field as a Cause of Dislocation Motion in $\mathrm{NaCl}$ Crystals. Journal of Experimental and Theoretical Physics Letters, 91, 91-95. https://doi.org/10.1134/S0021364010020086

[16] Buchachenko, A.L. (2001) Magnetic Isotope Effect: Nuclear Spin Control of Chemical Reactions. The Journal of Physical Chemistry A, 105, 9995-10003. https://doi.org/10.1021/jp011261d

[17] Buchachenko, A.L. (2013) Mass-Independent Isotope Effects. The Journal of Physical Chemistry B, 117, 2231-2238. https://doi.org/10.1021/jp308727w

[18] Buchachenko, A.L. (2006) Effect of Magnetic Field on Mechanics of Nonmagnetic Crystals: The Nature of Magneto-Plasticity. Journal of Experimental and Theoretical Physics, 102, 795-799. https://doi.org/10.1134/S1063776106050116

[19] Buchachenko, A.L. (2007) Magneto-Plasticity of Nonmagnetic Crystals in Microwave Fields. Journal of Experimental and Theoretical Physics, 105, 593-597. https://doi.org/10.1134/S1063776107090166

[20] Buchachenko, A.L. (2007) Physical Kinetics of Magneto-Plasticity. Journal of Experimental and Theoretical Physics, 105, 722-727. https://doi.org/10.1134/S1063776107100068

[21] Golovin, Yu.I., Morgunov, R.B., Ivanov, V.E., Zhulikov, S.E. and Dmitrievskii, A.A. (1998) Electron Paramagnetic Resonance in a Subsystem of Structural Defects as a Factor in the Plasticization of $\mathrm{NaCl}$ Crystals. Journal of Experimental and Theoretical Physics Letters, 68, 426-433. https://doi.org/10.1134/1.567884 
[22] Golovin, Yu.I., Morgunov, R. and Baskakov, A. (2002) Magneto-Resonant Softening of Solids. Molecular Physics, 100, 1291-1296. https://doi.org/10.1080/00268970110109763

[23] Chelidze, T., Gvelesiani, A., Varamashvili, N., Develidze, M., Chikhradze, V., Tchelidze, Z. and Elashvili, M. (2004) Electromagnetic Initiation of Slip: Laboratory Model. Acta Geophisica Polonica, 52, 49-62.

[24] Mubassarova, V.A., Bogomolov, L.B., Zakupin, A.S. and Panteleev, I.A. (2019) Acoustic Emission and Strain Responses of Rocks Triggered by Electromagnetic Action. Geosystems of Transition Zones, 3, 155-174. https://doi.org/10.30730/2541-8912.2019.3.2.155-174

[25] Weil, J.A. and Bolton, J.R. (2007) Electron Paramagnetic Resonance. Wiley, Hoboken. https://doi.org/10.1002/0470084987

[26] Zheng, Z. (2016) Research of Magnetic Bias Control System Based on STATCOM. World Journal of Engineering and Technology, 4, 9-15. https://doi.org/10.4236/wjet.2016.43D002

[27] Alshits, V.I., Darinskaya, E.V., Koldaeva, M.V. and Petrzhik, E.A. (2012) Anisotropic Resonant Magneto-Plasticity of $\mathrm{NaCl}$ Crystals in the Earth's Magnetic Field. Physics of the Solid State, 54, 324-331. https://doi.org/10.1134/S1063783412020059

[28] Alshits, V.I., Darinskaya, E.V., Morozov, V.A., Kats, V.M. and Lukin, A.A. (2013) Resonant Dislocation Motion in $\mathrm{NaCl}$ Crystals in the EPR Scheme in the Earth's Magnetic Field with Pulsed Pumping. Physics of the Solid State, 55, 2289-2296. https://doi.org/10.1134/S1063783413110024

[29] Alshits, V.I., Koldaeva, M.V. and Petrzhik, E.A. (2014) Low-Frequency Spectra of Dislocation Paths in $\mathrm{NaCl}$ Crystals in the Electron Spin Resonance Scheme. Journal of Experimental and Theoretical Physics Letters, 99, 82-88. https://doi.org/10.1134/S0021364014020039

[30] Alshits, V.I., Darinskaya, E.V., Koldaeva, M.V. and Petrzhik, E.A. (2016) Resonance Magneto-Plasticity in Ultralow Magnetic Fields. Journal of Experimental and Theoretical Physics Letters, 104, 353-364. https://doi.org/10.1134/S0021364016170045 